\title{
Supporting Information: The Effect of Host Incompatibility and Polarity Contrast on Ion Transport in Ternary Polymer-Polymer-Salt Blend Electrolytes
}

\author{
Bill K. Wheatle, Nathaniel A. Lynd, and Venkat Ganesan* \\ McKetta Department of Chemical Engineering, The University of Texas at Austin, Austin, \\ TX USA \\ E-mail: venkat@che.utexas.edu
}




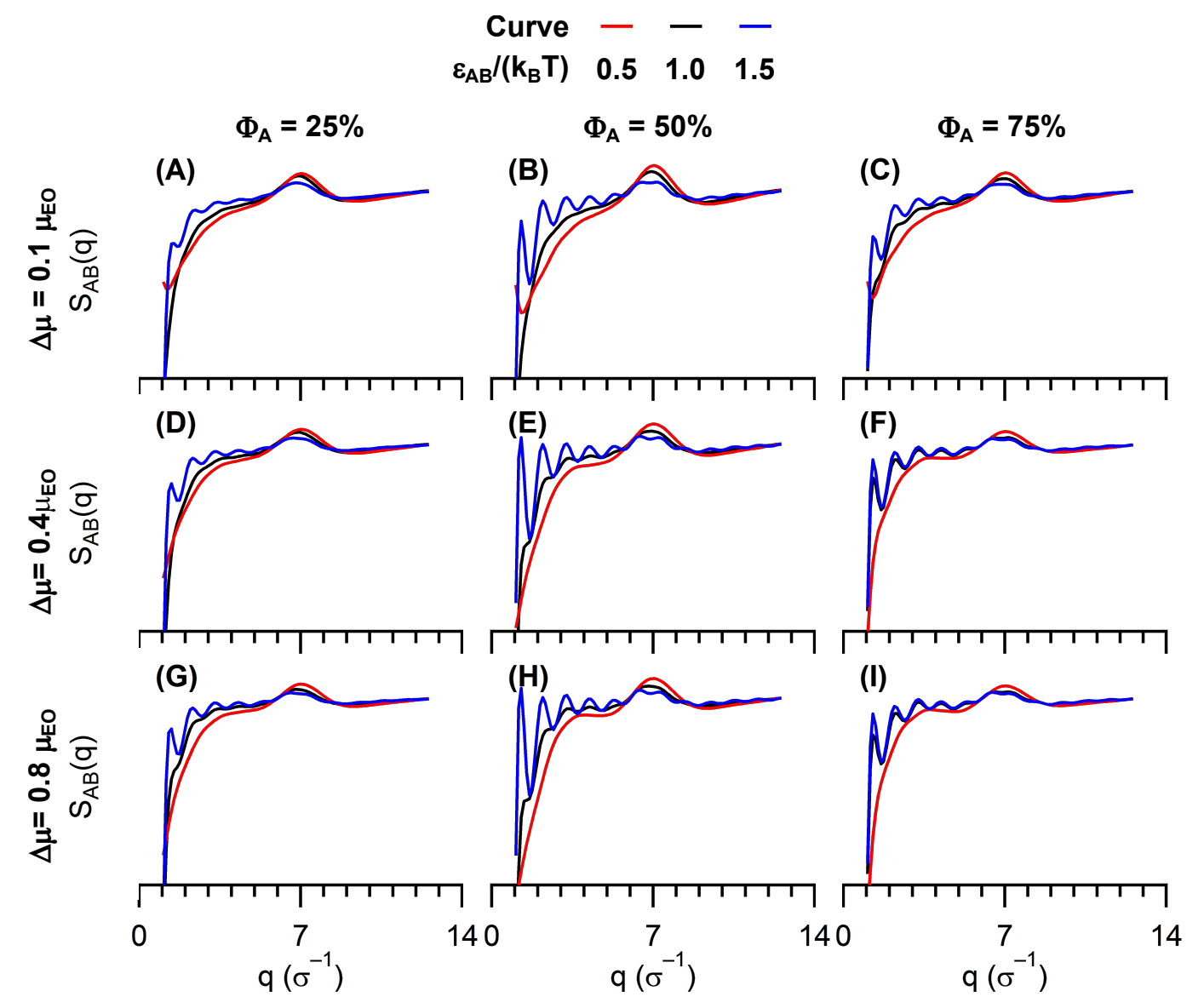

Figure S1: Structure factor $S_{A B}(q)$ as a function of polarity contrast $\Delta \mu / \mu_{E O}$, relative composition $\Phi_{A}$, and incompatibility $\varepsilon_{A B} /\left(k_{B} T\right)$. Note increasing sinusoid-like behavior arising in blends with increasing $\varepsilon_{A B} /\left(k_{B} T\right)$. 


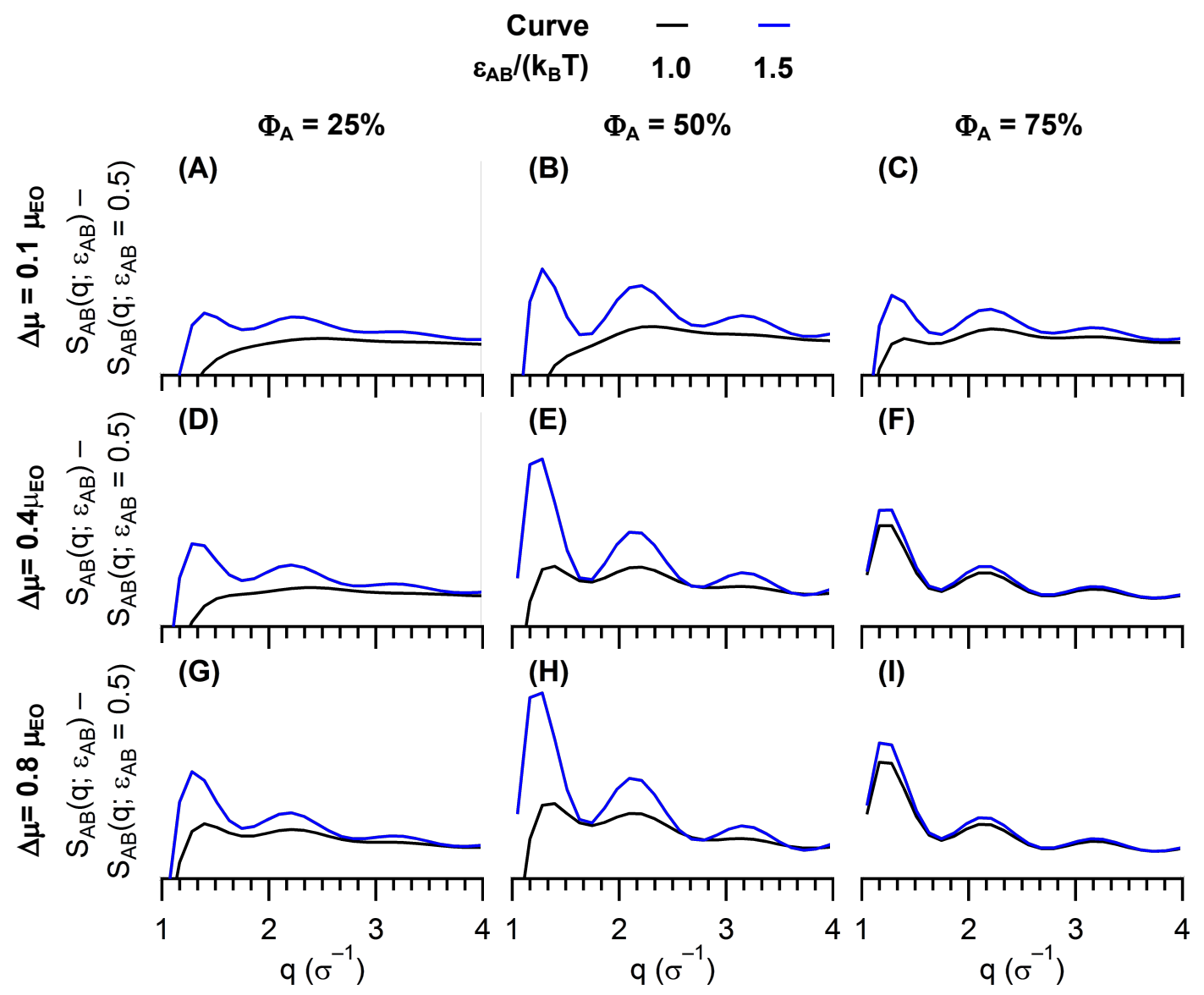

Figure $\mathrm{S} 2: S_{A B}\left(q ; \varepsilon_{A B} /\left(k_{B} T\right)\right)-S_{A B}(q ; 0.5)$ as functions of polarity contrast $\Delta \mu / \mu_{E O}$, relative composition $\Phi_{A}$, and incompatibility $\varepsilon_{A B} /\left(k_{B} T\right)$. Some "normalized" profiles retain their sinusoid-like behavior.

As noted in the main text of this work, we calculated the partial structure factor between polymers A and B $S_{A B}(q)$. Shown in Fig. S1, we can see see increasing sinusoid-like behavior arising in blends with increasing $\varepsilon_{A B} /\left(k_{B} T\right)$, for all $\Phi_{A}$ and $\Delta \mu / \mu_{E O}$. Such behavior is correlated with increased visually determined phase separation. We crudely "normalized" $S_{A B}(q)$ for higher values of $\Delta \mu / \mu_{E O}$ by that $\Delta \mu / \mu_{E O}=0.5$ and show the results of such an analysis in Fig. S2. Those simulations whose normalized $S_{A B}(q)$ profiles retain their sinusoid-like behavior are defined as being immiscible. 


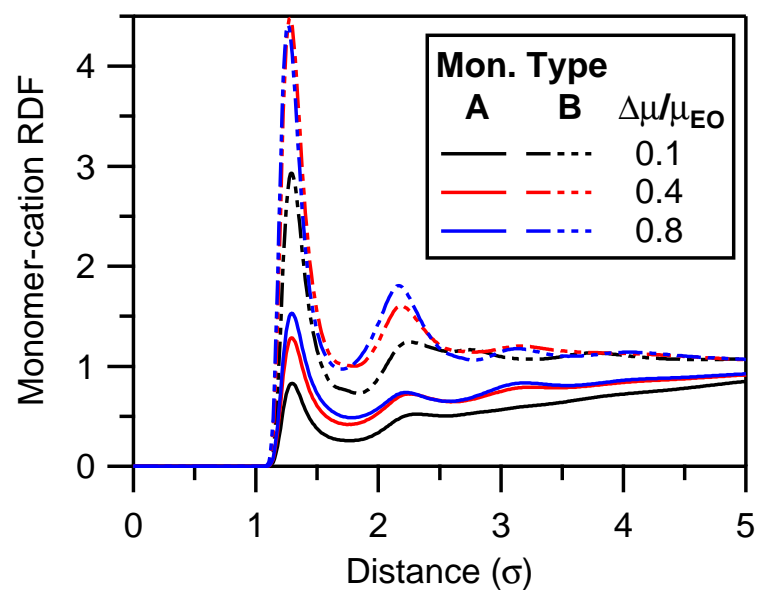

Figure S3: Radial distribution functions for $\Phi_{A}=50 \%$ between cations and low polarity polymer A beads (solid lines) and cations and high polarity polymer B beads (dashed lines) as functions of polarity contrast $\Delta \mu / \mu_{E O}$. Note the minima at approximately $1.8 \sigma$ and $2.75 \sigma$, which we denote to be the cutoffs for the first and second solvation shells. 


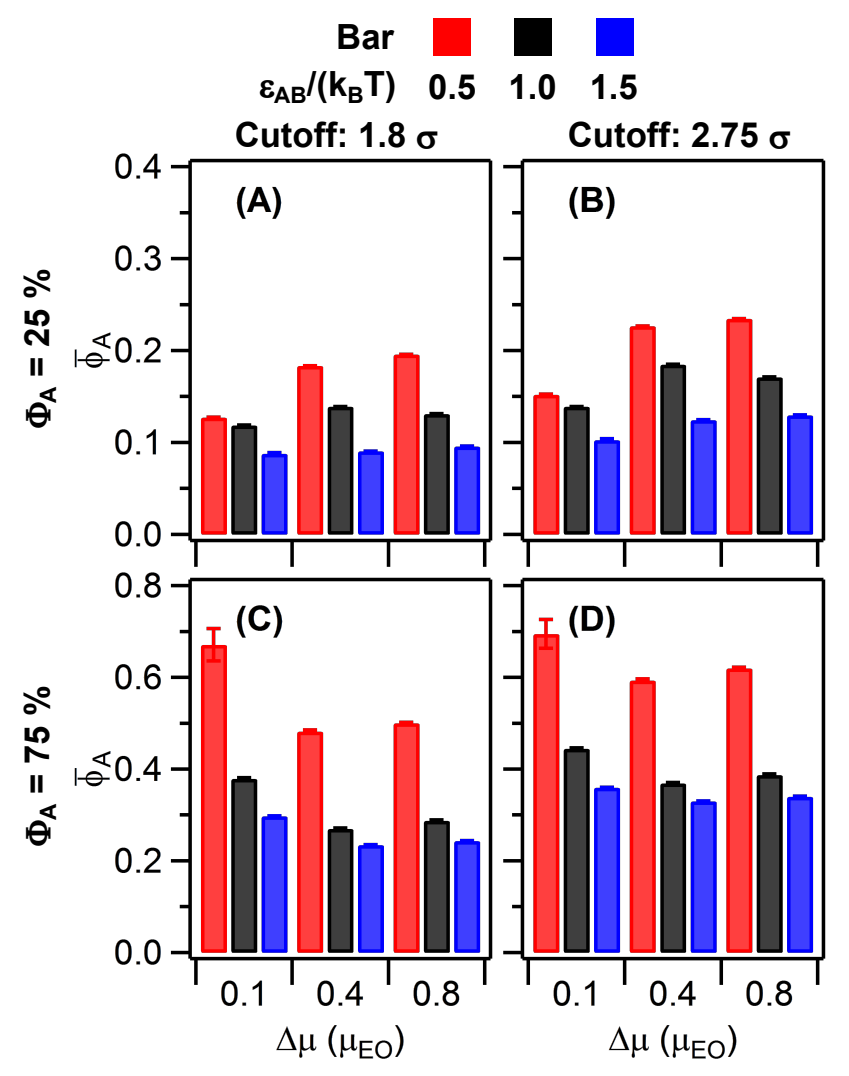

Figure S4: Average composition $\bar{\phi}_{A}$ in the first solvation shell and the sum of the first and second solvation shells around a typical cation as a function of polarity contrast $\Delta \mu / \mu_{E O}$, relative composition $\Phi_{A}$, and incompatibility $\varepsilon_{A B} /\left(k_{B} T\right)$.

As noted in the main text of this work, we used the radial distribution function between monomer and cation to determine the first and second solvation shells, shown in Fig. S3. We used these cutoffs to determine the average monomeric composition in a typical cation's first solvation shell and the combination of its first and second solvation shells. We present the results of such a calculation for $\Phi_{A}=25 \%$ and $\Phi_{A}=75 \%$ in Fig. S4. The results for $\Phi_{A}=50 \%$ can be found in the main text of the paper. Consistent with results in the main text of the paper, we can see that the high polarity polymer B favorably solvates cations in the first solvation shell, as $100 * \bar{\phi}_{A}<\Phi_{A}$. Consistent with our hypothesis, polymer A content increases we add the second solvation shell to our analysis, in some cases nearly reaching parity with bulk relative composition. Further, increasing solvent incompatibility $\varepsilon_{A B} /\left(k_{B} T\right)$ 
tends to decrease $\bar{\phi}_{A}$ in all cases, again consistent with our hypothesis.

$$
\begin{gathered}
\varepsilon_{\mathrm{AB}} /\left(\mathbf{k}_{\mathbf{B}} \mathbf{T}\right) \\
-0.5 \stackrel{-}{-}-1.0-1.5 \\
\text { Filled }=\text { Misc.; Hollow }=\text { Immisc. }
\end{gathered}
$$

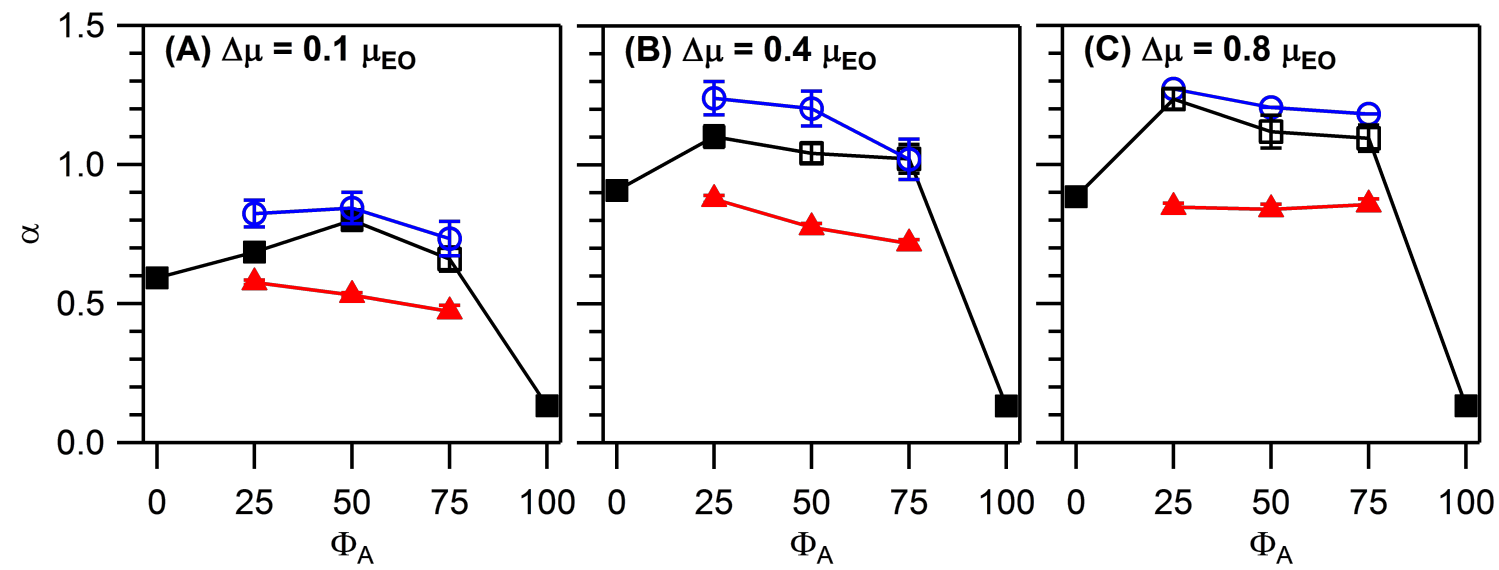

Figure S5: Degree of uncorrelated motion $\alpha$ as a function of polarity contrast $\Delta \mu / \mu_{E O}$, relative composition $\Phi_{A}$, and incompatibility $\varepsilon_{A B} /\left(k_{B} T\right) . \alpha$ increases with host incompatibility $\varepsilon_{A B} /\left(k_{B} T\right)$, independent of $\Delta \mu / \mu_{E O}$. 


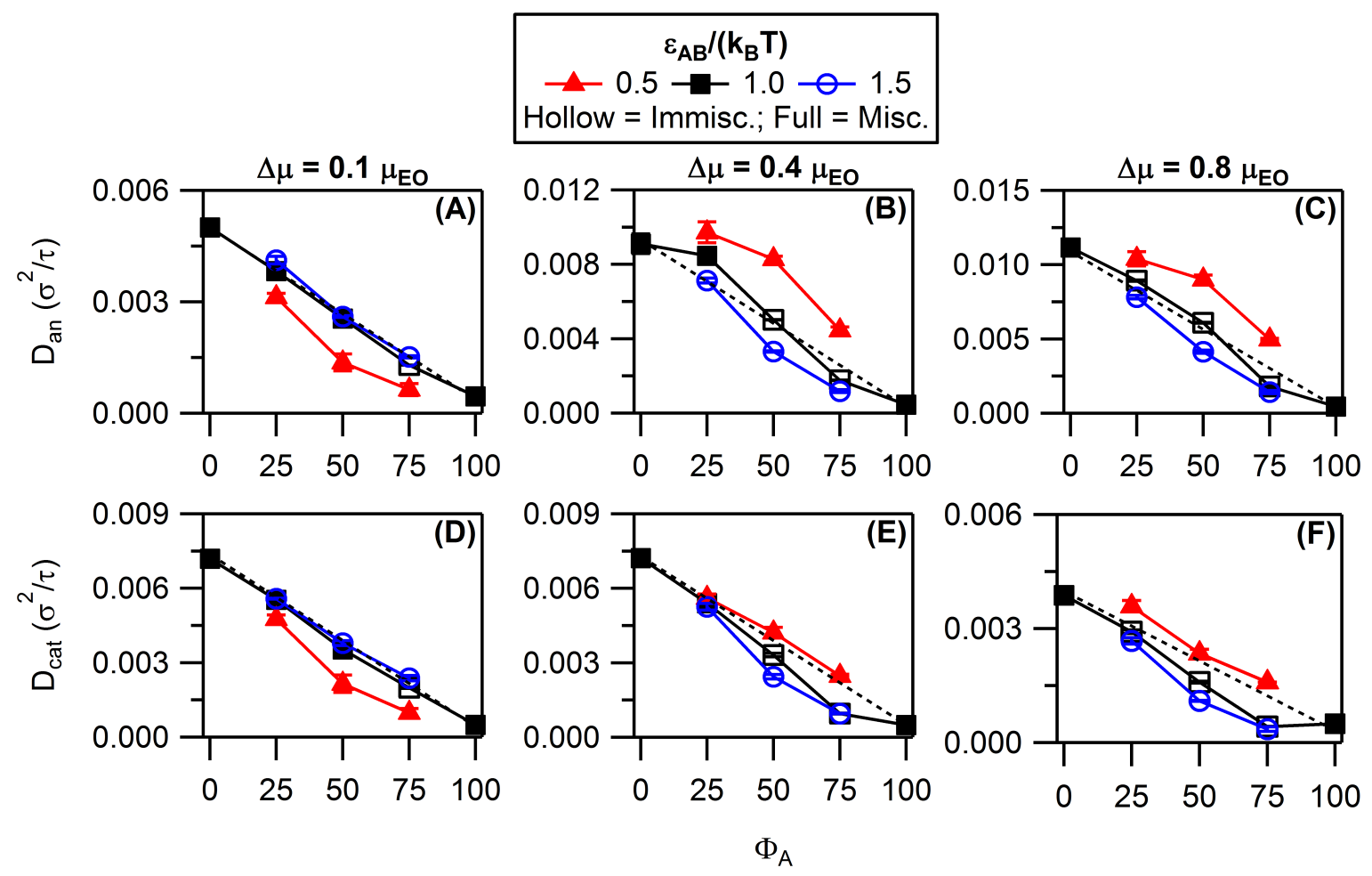

Figure S6: Ionic diffusion coefficients as functions of polarity contrast $\Delta \mu / \mu_{E O}$, relative composition $\Phi_{A}$, and incompatibility $\varepsilon_{A B} /\left(k_{B} T\right)$. The diffusion coefficients display similar behavior to conductivity in the main text of this work.

In the process of calculating the conductivity $\lambda$ for the main text of this work, we calculated the degree of uncorrelated motion $\alpha$ (see Fig. S5) and the ionic diffusion coefficients (see Fig. S6). We note that $\alpha$ increases with host incompatibility $\varepsilon_{A B} /\left(k_{B} T\right)$, independent of $\Phi_{A}$. However the diffusion coefficients more similarly to $\lambda$, which decrease with $\varepsilon_{A B} /\left(k_{B} T\right)$ at low $\Delta \mu / \mu_{E O}$ and increase with $\varepsilon_{A B} /\left(k_{B} T\right)$ at higher $\Delta \mu / \mu_{E O}$. 


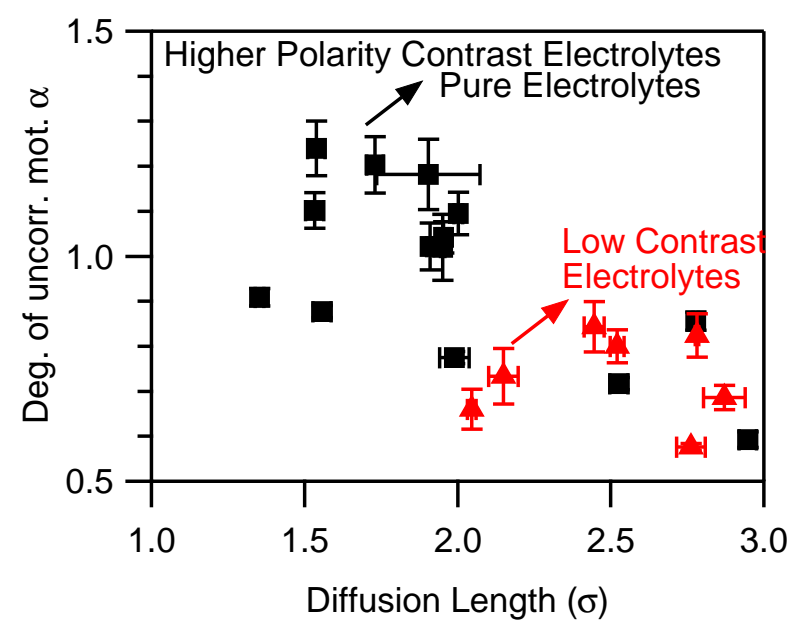

Figure S7: Degree of uncorrelated motion $\alpha$ as a function of cationic diffusion length $L_{c}$. Black squares represent pure electrolytes and mid- and high-polarity-contrast blend electrolytes, whereas red triangles represent low-polarity-contrast blend electrolytes. It can be seen that $\alpha$ is roughly inversely correlated with $L_{c}$ and that low-polarity-contrast electrolytes maintain a low $L_{c}$.

As discussed in the main text, we calculated the cationic diffusion length $L_{c}=\sqrt{6 D_{c} \tau_{C}}$. $\tau_{C}$ is the ion pair association time, calculated from the pair association autocorrelation function $C(t)$ :

$$
C(t)=\frac{\langle h(t) h(0)\rangle}{\left\langle h(0)^{2}\right\rangle}
$$

where $h(t)$ is unity if a cation and anion are separated by no more than $1.25 \sigma$ (the approximate minima of the anion-cation radial distribution function, see Fig. S3) and 0 otherwise. $C(t)$ was fit with a modified stretched exponential function: ${ }^{1,2}$

$$
C(t)=(1-\kappa) * \exp \left[-\left(\frac{t}{t_{i}^{*}}\right)_{i}^{\beta}\right]+\kappa
$$

The relaxation time $\tau_{C}$ was calculated, according to the following equation:

$$
\tau_{C}=\frac{(1-\kappa) t^{*}}{\beta} \Gamma\left(\frac{1}{\beta}\right)
$$


We plot $\alpha$ against $L_{c}$ in Fig. S7. As the error in $\tau_{C}$ was extremely large for two blend electrolytes $\left(\varepsilon_{A B} /\left(k_{B} T\right)=0.5, \Delta \mu / \mu_{E O}=0.1\right.$, and $\left.\Phi_{A} \in[50 \%, 75 \%]\right)$, we excluded from this figure. Otherwise, we can see that these two properties are roughly inversely correlated. For low-polarity-contrast electrolytes, depicted by the red triangles, $L_{c}$ remains short, seemingly allowing $\alpha$ to improve while ionic aggregation worsens, as discussed in the main text. 


\section{References}

(1) Williams, G.; Watts, D. C. Non-Symmetrical Dielectric Relaxation Behaviour Arising from a Simple Empirical Decay Function. Trans. Faraday Soc. 1969, 66, 80-85, DOI: $10.1039 / \operatorname{tf} 9706600080$.

(2) Williams, G.; Watts, D. C.; Dev, S. B.; North, A. M. Further Considerations of Non Symmetrical Dielectric Relaxation Behaviour Arising from a Simple Empirical Decay Function. Trans. Faraday Soc. 1971, 67, 1323-1335, DOI: 10.1039/TF9716701323. 